\title{
Elaborated integration of qualitative and quantitative perspectives in mixed methods research: a profound enquiry into the nursing practice environment
}

Keywords: integration of quantitative and qualitative perspectives, combining synthesised findings, nursing practice environment, elaborated integration in mixed methods

\begin{abstract}
Integration of quantitative and qualitative components in mixed methods research deserves more discussion in the literature, as it is critical for quality research outcomes. This paper describes an elaborated integration of these two components in the context of an empirical research study about nursing practice environments. Different types of integration such as combining notions at research conceptualization stage, mixing data collection methods and synthesizing findings, are described to show how qualitative and quantitative components can work interdependently to reveal "what" and "how" components of the research phenomenon. For example, through application of elaborated integration, it was revealed that "concern for cost" is a defining organizational characteristic of the current nursing practice environment. Additionally, we learnt about the "how" component with the revelation of nurses' belief that nurse administrators are acting with the intention of saving costs rather than enhancing quality of care. Therefore, this paper provides an empirical case of how to conduct integration of different components of mixed methods research with a great deal of depth, rationality and inquisitiveness.
\end{abstract}

\section{Introduction}

Mixed methods research has achieved good momentum in recent times. While the concept and practices of mixed methods research are still evolving, evidence such as an increase in mixed methods papers (Cameron, 2011a) and PhD theses (Plano Clark, 2010), suggest a wider application of mixed methods in different disciplines (Creswell, 2009a). Given such a groundswell for the use of mixed methods research, this is an appropriate time to discuss a critical aspect, that is, the integration of quantitative and qualitative components, inclusive of paradigms, methods, analysis techniques and findings, in mixed methods. At this stage, it should be clarified that authors of this paper are of the opinion that integration of quantitative and qualitative 
components in mixed methods involve conjoining the two different paradigms without compromising each paradigm’s unique proposition (Carroll \& Rothe, 2010).

In this paper we speak about integration, rather than triangulation for a reason. The view of triangulation taken here is that it can be a comprehensive methodology that enriches understanding of a research phenomenon through combination of different aspects, e.g. data sources, theories, investigators and methods (Al-Hamdan \& Anthony, 2010; Denzin, 1989). However, the term "triangulation" can draw misconception as sometimes it is advocated to combine different aspects mainly to enhance the validity of the research process and/or results (Greene, Caracelli, \& Graham, 1989; Lee \& Smith, 2012). On the other hand, the term “integration” by its dictionary meaning focuses on organizing the whole. As a research process, integration is well accepted for mixing quantitative and qualitative components of mixed methods so that these components become interdependent to develop a complete and profound picture of the research phenomenon (Bazeley, 2012; Woolley, 2009). Therefore, we favour the term "integration" because its connotation is closer to the agenda of this paper, that is, coalescing quantitative and qualitative components of mixed methods for better research outcomes. Firstly, we will explain what is meant by mixed methods research. Following, we will introduce elaborative integration before giving an example of how to apply it to a mixed methods study on nursing practice environments.

Mixed methods refer to a research method that "combines elements of qualitative and quantitative research approaches (e.g. use of qualitative and quantitative viewpoints, data collection, analysis, inference techniques)” (Johnson, Onwuegbuzie, \& Turner, 2007; p.123) for deeper understanding of the research phenomenon. Mixed methods can result in convergence or contradictions, but any of these outcomes will help the researcher in "superior explanation" (Johnson et al., 2007; p.115) of the research problem (Denzin, 1989; Johnson et al., 2007). It is important to note that achieving superior explanation that is, developing deeper understanding of different aspects of the research problem is definitely not an automatic process. Elaborative integration of quantitative and qualitative components can be a crucial prerequisite for this achievement. 
Exploring integration of different components in mixed methods research is a worthy topic, especially in the face of research practices that does not approach integration in full (Bazeley, 2012; Jang, McDougall, Pollon, Herbert, \& Russell, 2008; Johnson et al., 2007). One can comprehend elaborative integration from the following clarification from Woolley (2009):

Quantitative and qualitative components can be considered "integrated" to the extent that these components are explicitly related to each other within a single study and in such a way as to be mutually illuminating, thereby producing findings that are greater than the sum of the parts. (p. 7)

Recent literature (Brannen \& Moss, 2012; Frels \& Onwuegbuzie, 2013; Heyvaert, Maes, \& Onghena, 2013; Onwuegbuzie, 2012) encourage more elaborative integration in order to realise the potential of mixed methods research. One of the expectations is to better understand the mixed method research phenomenon with an integration that goes beyond mixing quantitative and qualitative data, but one that demonstrates a synthesis of the two different well-developed components (Heyvaert et al., 2013). The idea is to integrate in such a way that different components (e.g. qualitative \& quantitative) become interdependent throughout the research and generate an outcome that is greater than what would have been possible by any one of the component (Bazeley, 2010; Woolley, 2009). The interdependency of quantitative and qualitative components refers to one component helping the other in developing deeper understanding of the research problem (Carroll \& Rothe, 2010). Usually such dependency is built in mixed methods through frequent interactions at different points in the research process e.g. conceptualisation, analysis and theorising (Moran-Ellis et al., 2006, p. 46).

In line with the above literature of mixed methods research, this paper will use the term "elaborative integration" to refer to combining qualitative and quantitative components in depth. That is, reflecting on the interdependent roles of qualitative and quantitative components throughout the stages of conceptualisation of research to interpretation of research findings when rationally possible. Therefore, the highlights of the elaborative integration to be applied in this paper are; one) the mixing of the two components initiates from the research conceptualisation stage, and two) the mixing is not just happening between data collection methods but also with well- 
developed quantitative and qualitative findings (later on described as synthesized findings).

The paper will describe a specific research project within a nursing practice environment in New South Wales health services organisations. The aim is to exhibit steps that can facilitate elaborative integration in mixed methods research. Furthermore, this study provides empirical evidence of elaborative integration in mixed methods research that could guide future researchers towards better research outcome. There are three main sections in this paper: first, the method; which explains the quantitative and qualitative tools, procedures of data collection and profile of the respondents of the research. Second, a description of integration which communicates the steps of integration and associated rationales is provided. Third, the discussion on possible learning from the described elaborated integration will be addressed.

The Australian Nursing Federation (2009), in its report: Ensuring quality, safety and positive patient outcomes, claimed that a poor work environment contributes substantially to nurse turnover and deterioration in patient care (Armstrong, 2009). While improvement has been noted in the supply of the nursing workforce (World Health Organization, 2008, 2010), the challenge to support this workforce with a better nursing practice environment remains. Therefore this research which is part of a recently completed $\mathrm{PhD}$ study, was driven by the challenge of organising the nursing practice environment (NPE) so that the work environment is supportive to nurses and facilitates quality of care.

Accordingly, the research proposed to develop a profound understanding of the current nursing practice environment in New South Wales (NSW), Australia and identify areas for improvement. The research explored nurses' perception of relationships between three key aspects of nurses’ work and its context: organisational characteristics of the nursing practice environment, nurses' job satisfaction, and the quality of nursing care. The research questions that the thesis addressed were:

- What organisational characteristics define the current nursing practice environment in NSW health organisations? 
- $\quad$ How are the three domains of nursing practice environment, nurses' job satisfaction and quality of nursing care interrelated?

- How can the current nursing practice environment be improved?

The next section will provide relevant method information of the research about nursing practice environments in NSW, Australia.

\section{Methods}

In order to develop a comprehensive understanding of the nursing practice environment in NSW health services, the research applied mixed methods, including a quantitative survey questionnaire and qualitative one-on-one interviews with a twostage sequential design. The quantitative survey was conducted first and both the methods had equal status in this research (Author, 2011). In line with mixed method conventions, this research design sits closest to the typology of "partially mixed sequential equal status design” (Leech, \& Onwuegbuzie, 2009, p. 270). One point of differences between the chosen typology and the research design is the timing of mixing the methods. Leech \& Onwuegbuzie (2009) described the typology to mix methods at the stage of data interpretation, but in this research the mixing of methods was initiated earlier during the research process. The mismatch with the typology is not worrying, since typologies can sometimes be inadequate to capture practical complexities in research designs (Guest, 2013).

\section{Quantitative and qualitative tools}

The quantitative data collection tool, the survey questionnaire, contained 63 items, each item had one to seven Likert scale, to measure the domains of nursing practice environment, nurses' job satisfaction and quality of nursing care utilising adapted quantitative instruments of Lake (2002), Hinshaw and Atwood (1985) and Greenslade and Jimmieson (2007) respectively. The main criterion in instrument selection was that the chosen instruments were theoretically relevant to the research context and purpose. To ensure the relevance of the survey questionnaire to the Australian context, the chosen instruments were taken through an adaptation process, comprising of steps such as peer review, pilot study and statistical test of validity and reliability (Siddiqui \& Fitzgerald, 2009). The survey questionnaire addressed the first 
two research questions; however, the analysis of the survey data was necessary to answer the third research question as well.

The qualitative data collection tool, the semi-structured, one-on-one interviews $\mathrm{f}$ were organised around three overarching questions. These questions permitted exploration of all the three research questions in the thesis. The three questions were; i) "Do you agree with the survey findings?" "How would you describe the existing condition of your work environment?" ii) "We have been talking about the three factors; nursing work environment, nurses’ job satisfaction and quality nursing care. Do you think these three are related?" and iii) "If you would like to improve your work environment, what areas should be worked upon?". The second overarching question was influenced by the findings of the earlier conducted survey questionnaire. It should be noted that while the interviews were organised around the overarching questions, considerable flexibility was also kept to allow the interview to vary in scope and depth (Fylan, 2005). For example, probing questions related to the survey findings were discussed only if the participants raised the topic.

\section{Procedures of data collection}

The data collection took place in two phases in 2009. During phase one a survey questionnaire was administered. About four months after the survey, phase two consisted of the semi-structured interviews. The Nursing \& Midwifery Board (NMB), NSW approved a request for assistance, in recruiting participants for the survey questionnaire. Subsequent to approval from the NMB, ethical approval was also received from the Human Research Ethics Committee of the University of Western Sydney in Australia (protocol no. H6460).

Participants in this study were recruited via purposive sampling from the register of the Nursing and Midwifery Board (NMB), NSW. The respondents were required to be practicing nurses and have a minimum of two years of nursing experience in an inpatient practice environment in NSW health service organisations. The registrar at NMB circulated survey packs in hard copy, to 2050 nurses at their residential addresses. A total of 157 packs were returned, achieving a response rate of $7.6 \%$. The 
final survey sample size was 136, since 21 respondents failed the screening criteria as they were nurses who either are currently not working in nursing or working in nursing but outside of NSW. The 7.6\% survey response rate is accepted as a major limitation of this study. Accordingly, the study does not aim to represent a generalised picture of the nursing practice environment in NSW health service organisations.

A return of the questionnaire was deemed to be implied consent for voluntary participation. Sixty five respondents self-nominated for an interview, from the survey sample of 136, by returning the signed interview consent forms, which they received along with the survey questionnaire. Out of the pool of 65 self-nominated nurses, interviewees were selected according to purposive sampling procedure of maximumvariation type (Kuper, Lingard, \& Levinson, 2008; Teddlie \& Yu, 2007). Therefore, the intention was to include a variety of clinical practice areas (e.g. medical, critical care, per-operative) and nursing classifications, that is, clinical nurse consultants, nurse managers, clinical nurse educators, clinical nurse specialists and registered nurses. Moreover, nurses belonging to the clinical practice areas that employed the majority of nurses in NSW (at the data collection time) were given priority in the screening process. Applying the criteria of clinical practice areas and nursing classifications, 21 nurses were selected for interview. Nurses from such a variety of backgrounds were expected to provide broad and comprehensive answers to the interview questions.

\section{Research respondents}

This study represented a sample of 136 survey respondents and 21 interviewees. In the survey sample, more that $70 \%$ belonged to public hospitals and 95\% worked in a hospital type work environment. The survey respondents came from clinical practice areas of; medical/surgical (23.6\%), critical care (18\%), midwifery (10.6\%), mental health (8.9\%), aged care (8.1\%), peri-operative (7.3\%) and others (23.5\%). Ninety two percent of them are females, with an average age of 50. The nursing classification composition of the survey sample is: registered nurse (61\%), nurse manager (15\%), clinical nurse specialists (13\%), clinical nurse consultant (7\%), clinical nurse educators (2\%) and nurse educators (2\%). From the 21 interviewees, the majority (17) worked at public hospitals and belonged to a variety of practice areas such as mixed medical, critical care, aged care, midwifery and pediatrics. The nursing classification 
of the interviewees are: bedside/registered nurses (7), clinical nurse consultants (3), clinical nurse specialists (2) and clinical educators (2). The interviewees' age ranged from 39 to 63 years.

The next section will illustrate how elaborated integration was conducted in the above mentioned research.

\section{Description of Integration}

Mixed methods research can be integrated by employing frequent and rational interactions between the two components. For example, when research questions are rationally drawn, allowing interaction of the two components, then the likely outcome is that the quantitative and qualitative components will become interdependent to develop deeper understanding of the research phenomenon. Another crucial understanding for mixed methods researchers is that the researcher's own guiding principles are concomitant to integrations that are possible. The literature defines guiding principles as a basic set of beliefs that guide research actions (Creswell, 2009b; Guba, 1990). Hence, before describing the steps of integration, the guiding principles of the researchers in this research about nursing practice environment are explained.

The researchers believed that their role was to generate knowledge, in an ethical manner, through interpretation of practical nursing experiences that answered the research questions. From this viewpoint, they could relate to the epistemology of pragmatism where knowledge is viewed not as a representation of reality, but rather as a tool to develop "a more or less satisfactory relation with the world, knowledge can be evaluated for whether it works for us in relation to a particular goal or interest" (Cornish \& Gillespie, 2009; p. 807). Due to such epistemological positioning, this study about nursing practice environment could avail multiple theoretical frameworks, from quantitative and qualitative perspectives and multiple disciplines, for example, organisational behavior, management, and/or sociology. The main criteria were that the frameworks had to be relevant to the research questions and conducive to generate knowledge of theoretical and pragmatic value. It might also be important to clarify that the objective of comprehensive answer to research questions 
does not mean that the researchers were seeking to generalise the findings to all nursing experiences in Australia. Rather, the expectation was that interaction of data collection methods will uncover greater insights, providing practical value for nurses at a specific time and within a specific environment. As such, this mixed method sought for insights which are -at best- transferable to other contexts.

In line with the above mentioned guiding principles, the integration of quantitative and qualitative components in this paper occurred mostly in three ways; combining the research conceptualization notions, combining the data collection methods and combining the synthesized findings. The subsequent section will describe each of these ways of integrations in detail.

\section{Integration I: Combining the research conceptualization notions}

Notions at research conceptualization stage refer to the thoughts that researchers started with and applied to initial research stages e.g. defining research problem, developing research questions and deciding tools to be used. These thoughts are tied to the researchers' opinion that quantitative and qualitative paradigms can be integrated without compromising each paradigm's unique proposition, since the integration reveals different elements of the research phenomenon (Carroll \& Rothe, 2010), that is, "what" (from quantitative) and "how" (from qualitative component).

In this research of nursing practice environment in health service organisations, the integration of qualitative and quantitative notions took place from the very beginning. Even in the stage of defining the research problem and research questions, researchers were keen to compile a comprehensive picture of the nursing practice environment from both components. In line with that interest, the research problem of "developing a better understanding of the nursing practice environment and to recommend ways for its improvement" was broken down into research questions that had both quantitative and qualitative elements to it. The first research question is: "what organisational characteristics are defining the current nursing practice environment?” This question has a quantitative element to it. It is about a measurement of specific characteristics within an organization, i.e. cultural values, nurse manager ability, nurse participation in hospital affairs, nursing foundations for quality of care, staffing 
and resource adequacy and nurse-doctor relations. This question was investigated with previously validated research instruments (Lake, 2002; Schmalenberg \& Kramer, 2008) that measure the organisational characteristics within established theoretical frameworks. However, the question also reveals a qualitative element. It clearly asked for perceptions of organisational members about the organisational characteristics within respondents' own working environment. As such an interpretative framework was also necessary to come to any conclusions. The answers to the first research question were only deemed comprehensive when both the quantitative and qualitative components of the current nursing practice environment were measured through aggregated data analysis, as well as authenticated by coalface workers through their story telling of their workplace.

This view of mixed elements also applied to the second research question, which is; “How are the three domains of nursing practice environment, nurses' job satisfaction and quality of nursing care interrelated?” The second research question needed to seek a quantitative assessment, in addition to qualitative rationale behind the relationship of the nursing practice environment and the other two domains. Similarly, the third research question, "How can the current nursing practice environment be improved”, is dependent on quantitative identification of areas needing improvement that came from the analysis of quantitative survey data. Moreover, the third research question required to uncover possible directions towards such improvement from qualitative insights from nurses. Therefore the rationale behind the explained interaction was that the three research questions represented integrated queries, which involved quantitative elements as "what" and qualitative elements as "how".

The other significant interaction between qualitative and quantitative notions occurred while adapting existing quantitative instruments (e.g. practice environment scale nursing work index for measurement of nursing practice environment by Lake, 2002) for the survey. In this adaptation process, steps such as peer review and pilot study were conducted to adapt the instruments to the Australian context in regards to language, content and structure. These steps reflected qualitative notion as it collected opinions in open text boxes from nursing researchers and practitioners about the suitability of the instrument for the Australian nursing practice environment. These steps ensured that the survey had content validity, that is, the content of the survey is 
consistent with the established concepts of the Australian nursing practice environment (Fitzpatrick, 1983). Additionally, the adaptation process applied confirmatory factor analysis and reliability tests to ensure that the survey data reflected valid constructs of nursing practice environment in quantitative terms (Angoff, 1988; Chiang, \& Lin, 2009). Hence, the interaction of qualitative and quantitative perspectives during the adaptation of the survey instrument enhanced the credibility of the research process. One example outcome of the interactions at this stage is that a question about continuity of care (same nurse/patient one day to next) was dropped from the survey. The question received a low factor loading score in the quantitative test. Moreover, the qualitative feedback indicated that nurses could not relate to this question since shortage of resources makes it hard to assign the same nurse to the same patients.

\section{Integration II: Combining data collection methods}

The two data collection methods employed in this study are survey questionnaire and semi-structured interviews. The interviews were conducted about four months post the survey period. Due to such sequencing, the survey findings could partially guide the interview format and thus, allowed to integrate the two data collection methods.

The interviews began by reporting a summary of the findings from the survey, that is, the scores on nursing practice environment and a request to disclose whether interviewees' perception about their own practice environment matches or contradicts with the rating of the survey. This introduction provided a context for posing an overarching question about participants' feelings about their current nursing practice environment and to open up a discussion about the research questions. The second overarching question in the interview, an inquiry about the interrelation between nursing practice environment and nurses' job satisfaction and quality of nursing care, is closely tied to the survey findings that indicated a possible relationship between the three domains. Thus the question was a direct prompt to collect further insights on this matter through participants' stories. Such interaction between quantitative and qualitative methods at the interview stage, which is not unusual in mixed methods (Leech \& Onwuegbuzie, 2009), was intended to facilitate a deeper examination of the second research question in the study. 
Moreover, content of some of the probing questions was intended to cover topics of unusual findings in the survey. An example of such topic is the survey finding of a relatively weak association between the characteristics of nurse participation in hospital affairs and quality of nursing care. Accordingly, during the interviews probing questions were put to interviewees to reflect firstly on their understanding of participation in hospital affairs and secondly on whether interviewees find participating in hospital affairs contribute to delivery of quality care. However such probing questions were covered in the interview, only if the topics were raised by the interviewees.

It should be noted that the above mentioned interactions between data collection methods were relevant for few reasons. One, the research questions investigated during the survey and the interviews were interrelated. Two, as mentioned in the method section, the participants for the interviews were selected from the sample pool of survey participants. Therefore, the interview participants had some orientation with the survey topic and could relate to it.

\section{Integration III: Combining synthesized findings}

This set of integration took place while answering the research questions from the combination of synthesized findings. Importantly, integration at this stage involved merging the synthesized findings into a new data set for further analysis. Synthesized findings are raw data turned into findings through application of analysis techniques of quantitative and qualitative types such as regression and thematic analysis respectively. It is important to note that synthesized findings could be generated in this study because the surveys and the interviews were supported with distinctive data collection and synthesis processes. In the next paragraph, a brief description of the process of individual synthesis is provided. Such description is necessary to depict how synthesized findings are more explanatory and once integrated, can facilitate better understanding of the research problem in creative manners (Bazeley, 2009; J.C. Greene, 2007; Heyvaert et al., 2013). 
The survey questionnaire was structured and initially analysed, using the theoretical framework of an extended magnet study (Lake, 2002) and extended structure, process and outcome (SPO) paradigm (Mitchell, Ferketich, \& Jennings, 1998). In line with the deductive nature of quantitative perspective, the initial analysis of the survey data was organised to extract answers to the first two research questions. Quantitative techniques e.g. mean rank, Spearman rank-order correlations and mediator analysis through regression were applied to synthesize the survey data. Few findings from the synthesized survey data are: characteristics (e.g. cultural values, nurse participation in hospital affairs) of nursing practice environment are correlated. Furthermore, there can be a reciprocal relationship between nursing practice environment, nurses' job satisfaction and quality of nursing care.

On the other hand, a different set of frameworks, that is, the social exchange theory (Blau, 1986) and four worlds of hospital (Glouberman \& Mintzberg, 2001a, 2001b) were found relevant in analysing the interview data. In line with the inductive nature of a qualitative perspective, the interview data were synthesized through thematic analysis. The main step in the thematic analysis was the comparison of patterns of commonality and divergences in nurses' experiences (Boyatzis, 1998). The iterative process of thematic analysis ultimately synthesized the interview data into eight themes. These themes reflected different aspects of nursing experiences (e.g. availability of resources, sense of fulfillment, interactions with nurse administrators, suggestions to improve current work environment) in their nursing practice environment.

Once the above mentioned synthesised quantitative and qualitative findings were ready and a new combined dataset was analysed, integration occurred in the process of reaching an overall answer to each of the three research questions. The stage involved developing a combined picture of nursing practice environment, putting the qualitative and quantitative findings together for a third analysis. For example, the survey finding had identified "concern for cost" in the cultural values to be a defining organisational characteristic in the nursing practice environment. The interview findings further disclosed that nurses believed, nursing administrators are enforcing changes in their workplaces in order to control costs rather than to enhance patient 
care. Hence, the integration of synthesised findings provided greater description of the "what" and "how" in answering the research questions.

Interestingly, the interpretation of blended synthesised findings also allowed to combine the theories of a quantitative perspective (extended magnet study, extended SPO paradigm) and qualitative one (social exchange theory and the four worlds of hospital). This point needs to be explained with specific example. As mentioned earlier, one of the findings from the quantitative survey within the theoretical framework of extended magnet study is that conflict of care and financial control is a defining characteristic in the current nursing practice environment. Upon analysis of qualitative interviews, it was seen that this finding resonates with the theoretical framework of the four worlds hospital (Glouberman \& Mintzberg, 2001a). Glouberman and Mintzberg (2001a) referred to conflict of care and financial control between two worlds, that is, the world of care of nurses and the world of control of administrators. However, great value addition resulted when these synthesised findings were analysed in combined theoretical frameworks. Both the theories conveyed that conflict of care and financial control has long-term damaging effect in health service delivery, which is a value addition since it reiterated the necessity to address the conflict. The other value addition came from the differences between the two theoretical frameworks. The framework of four worlds of hospital had highlighted the distinctive focus of different roles (e.g. doctors, nurses, administrators/managers) whereas the extended magnet study only covered the nursing roles. The insight of exchanges between multiple roles was important to understand as the conflict that Glouberman and Mintzberg (2001a) had identified between nurses and administrators (between two worlds) also exists within nurses, that is, between nurse clinicians and nursing administrators. This is a value addition because it revealed the need to better integrate the role of nursing administrator into the world of nursing.

\section{Discussion}

Integration in mixed method research is a crucial step to harness quality research outcomes. Integration facilitates interdependent roles between qualitative and quantitative perspectives, generating stimulating imaginations regarding the research 
problem (Brewer \& Hunter, 2006; J.C. Greene, 2007; Onwuegbuzie, 2012). Given the significance of integration in mixed methods research, it is concerning that the topic is yet to receive due attention from researchers as evidenced by inadequate depth in practices and discussions of integrations (Bazeley, 2010; Bryman, 2008; Heyvaert et al., 2013) .

Reflecting on the above mentioned concern, the paper explained how a research into nursing practice environment in NSW conducted an elaborated integration. The three ways of integration i.e. combining the notions at research conceptualization stage, the data collection methods and the synthesized findings, as explained in this paper, can offer insights into the integration component of "Praxis", application of theory in practices of mixed method research approaches and design (Cameron, 2011b; P.102 ) for future mixed methods research. For instance, the explanation of integration of qualitative and quantitative findings, in this study, articulated an example of the theme of "mixed method research at synthesis level (MMRS)" (Heyvaert et al., 2011; p.662). The prime requirements of MMRS are: well-developed (e.g. sizeable sample base, rigorous data collection, meaningful inferences) individual qualitative and quantitative strands (Creswell \& Tashakkori, 2007) and linkage between these two strands for better understanding of the research phenomenon (Bryman, 2007). In line with the requirements of MMRS, this paper narrated how specific steps such as connecting theories of quantitative and qualitative components generated greater insight on nursing practice environment.

This paper explained rationales and benefits of integration in empirical form and resonated with the notion that integration in mixed methods is a rational, yet interpretive process. Malina, Hanne and Selto (2011) advised that mixed methods research is less traditional and therefore can be more inquisitive. Drawing on the experience of mixed methods research into nursing practice environment, the paper articulates that one major area of learning for mixed method researchers is the balance between rationality and the inquisitiveness that can "challenge conventions" (Bazeley, 2009; Malina et al., 2011). The research into nursing practice environment adhered to rationality by allowing the integrations to be influenced by researchers' guiding principles. Rationality was also evidenced during the interviews, where the probing queries from the survey were discussed only if the interviewees raised the topics. 
Such rationality avoided compromising the inherent nature of qualitative method (i.e. semi-structured interview in this case) by the influence of the quantitative method (i.e. survey in this case) (Pearce, 2012).

Then again, in the research into nursing practice environment, we took a critical look at conventions on a few occasions and found these occasions to be rewarding. During the combination of quantitative and qualitative findings (as mentioned in section of combining the synthesized findings ), we questioned the proposition of Glouberman \& Mintzberg (2001a) that nurses are bonded together in one nursing world with their focus on patient care. The exercise revealed insights that nurses can function differently depending on their focus on patient care, generating conflicts of care and financial control even within the nursing world. Similarly, while combining the data collection methods, interviewees were given the scope to contradict or confirm the survey findings. The effect of which was to have another critical look at the survey findings in the presence of realistic interview findings. Consequently, the research was rewarded with further insight that nursing practice environment is not only a construct of organisational characteristics, such as cultural values, staffing and resource adequacy, but also a facilitator of exchanges between people in health services organisations.

Another associated thought on the learning of balancing act between rationality and inquisitiveness, is that integration in mixed methods demand competency with each of the perspectives of qualitative, quantitative and the combined one. Recently, researchers (Onwuegbuzie, Frels, Collins, \& Leech, 2013) have disclosed a four phase model (i.e. conceptual, technical, applied \& emergent scholar) for mixed methods research course in which researchers are trained in skills of each of the qualitative and quantitative perspectives to facilitate a combination of perspectives. We also know that the training for mixed methods research is most effective when researchers learn about quantitative and qualitative perspectives in non-biased manner (Bazeley, 2003). All these insights, along with the ones presented in this paper, can assist with the generation of more mixed methods research that reflects elaborated integration.

On the basis of above discussion, it can be stated that this paper attempted contributing to pragmatic knowledge for mixed methods. First; the paper has endorsed the overarching learning that mixed methods will lose its potential without 
elaborative integration built into it. Second, through the articulation of MMRS, the paper has resonated that integration in mixed methods can be a rational, yet interpretive process. Third, the paper has professed that integration in mixed methods would require researchers to appreciate the unique proposition of different paradigms and develop research competencies across the paradigms.

\section{Conclusion}

Depending on the questions posed, ideally the integration of qualitative and quantitative perspectives in mixed methods research should be elaborative, reflecting interdependent roles between the perspectives. This paper has provided empirical evidence of how elaborated integrations in mixed methods research can achieve quality research outcomes. Researchers are urged to realise the potential of mixed methods research by conducting integrations of qualitative and quantitative data and findings with great depth, rationality and inquisitiveness. 


\section{References}

Al-Hamdan, Z., \& Anthony, D. (2010). Deciding on a mixed-methods design in a doctoral study. Nurse Researcher, 18(1), 45-56.

Angoff, W. H. (1988). Validity: An Evolving Concept. In H. Wainer \& H. Braun (Eds.), (illustrated ed., pp. 267). Test Validity: Lawrence Erlbaum Associates.

Armstrong, F. (2009). Ensuring quality, safety and positive patient outcomes: Why investing in nursing makes sense. In R. Elizabeth (Ed.). Melbourne: Australian Nursing Federation

Author. (2011). Caring for all: A mixed methods study into the nursing practice environment and its relationship with nurses' job satisfaction and quality of nursing care in NSW health service organisations. (Doctor of Philosophy), University of Western Sydney, Sydney.

Bazeley, P. (2003). Teaching mixed ethods. Qualitataive research journal, 3, 117126.

Bazeley, P. (2009). Editorial: Integrating Data Analyses in Mixed Methods Research. Journal of Mixed Methods Research, 3(3), 203-207. doi: $10.1177 / 1558689809334443$

Bazeley, P. (2010). Computer-Assisted Integration of Mixed Methods Data Sources and Analyses. In A. Tashakkori \& C. Teddlie (Eds.), Handbook of mixed methods in social \& behavioral research (Vol. 2nd). LA: Sage Publications.

Bazeley, P. (2012). Integrative Analysis Strategies for Mixed Data Sources. American Behavioral Scientist, 56(6), 814-828. doi: $10.1177 / 0002764211426330$

Blau, P. M. (1986). Exchange and power in social life. New Jersey Transaction Publishers (Original work published in 1964 by Wiley \& Sons Inc., New York).

Boyatzis, R. E. (1998). Transforming qualitative information: thematic analysis and code development: Sage Publications Inc. Thousand Oaks, CA.

Brannen, J., \& Moss, G. (2012). Critical Issues in Designing Mixed Methods Policy Research. American Behavioral Scientist, 56(6), 789-801. doi: $10.1177 / 0002764211433796$

Brewer, J., \& Hunter, A. (2006). Foundations of multimethod research: Synthesizing styles (Vol. 2nd ed. ). Thousand Oaks, CA: Sage.

Bryman, A. (2007). Barriers to Integrating Quantitative and Qualitative Research. Journal of Mixed Methods Research, 1(1), 8-22. doi: $10.1177 / 2345678906290531$

Bryman, A. (2008). Why do Researchers Integrate/Combine/Mesh/Blend/Mix/Merge/Fuse Quantitative and Qualitative Research? In M. M. Bergman (Ed.), Advances in mixed methods research (pp. 87-100). Thousand Oaks, CA: SAGE.

Cameron, R. (2011a). Mixed methods in business and management: A call to the 'first generation'. Journal of Management and Organization, 17(2), 245267.

Cameron, R. (2011b). Mixed methods research : the Five Ps framework. Electronic Journal of Business Research Methods, 9(2), 96-108. 
Carroll, L. J., \& Rothe, J. P. (2010). Levels of Reconstruction as Complementarity in Mixed Methods Research: A Social Theory-Based Conceptual Framework for Integrating Qualitative and Quantitative Research. International Journal of Environmental Research and Public Health, 7(9), 3478-3488.

Cornish, F., \& Gillespie, A. (2009). A Pragmatist Approach to the Problem of Knowledge in Health Psychology. J Health Psychol, 14(6), 800-809. doi: $10.1177 / 1359105309338974$

Creswell, J. W. (2009a). Editorial: Mapping the Field of Mixed Methods Research. Journal of Mixed Methods Research, 3(2), 95-108. doi: $10.1177 / 1558689808330883$

Creswell, J. W. (2009b). Research Design: Qualitative, Quantitative and Mixed Methods Approaches (3rd ed.) (3rd edition ed. Vol. 3rd edition). Los Angeles: Sage Publications.

Creswell, J. W., \& Tashakkori, A. (2007). Editorial: Differing Perspectives on Mixed Methods Research. Journal of Mixed Methods Research, 1(4), 303308. doi: $10.1177 / 1558689807306132$

Denzin, N. (1989). The Research Act: A Theoretical Introduction to Sociological Methods (3rd ed.). Englewood Cliffs, NJ: Prentice Hall.

Fitzpatrick, A. R. (1983). The meaning of content validity. Applied Psychological Measurement, 7(1), 3-13.

Frels, R. K., \& Onwuegbuzie, A. J. (2013). Administering Quantitative Instruments With Qualitative Interviews: A Mixed Research Approach. Journal of Counseling and Development : JCD, 91(2), 184-194. doi: 10.111 l/j.l7520606.2001.tb01 162.x

Fylan, F. (2005). Chapter 6: Semi-structured interviewing. In J. Miles \& P. Gilbert (Eds.), A Handbook of Research Methods for Clinical and Health Psychology New York, USA: Oxford University Press.

Glouberman, S., \& Mintzberg, H. (2001a). Managing the Care of Health and the Cure of Disease - Part II: Integration. Healthcare Management Review, 26(1), 70-84.

Glouberman, S., \& Mintzberg, H. (2001b). Managing the Care of Helath and the Cure of Disease - Part I: Differentiation. Health Care Management Review, 26(1), 56-69.

Greene, J.C. (2007). Mixed methods in social inquiry. San Francisco: Jossey-Bass.

Greene, J.C, Caracelli, V. J., \& Graham, W. F. (1989). Toward a Conceptual Framework for Mixed-Method Evaluation Designs. Educational Evaluation and Policy Analysis, 11(3), 255-274. doi: 10.3102/01623737011003255

Greenslade, J. H., \& Jimmieson, N. L. (2007). Distinguishing between task and contextual performance for nurses: development of a job performance scale. Journal of Advanced Nursing, 58(6), 602-611.

Guba, E. (1990). The paradigm dialogue. Newbury Park, CA: Sage Publications.

Guest, G. (2013). Describing Mixed Methods Research: An Alternative to Typologies. Journal of Mixed Methods Research, 7(2), 141-151. doi: $10.1177 / 1558689812461179$

Heyvaert, M., Maes, B., \& Onghena, P. (2013). Mixed methods research synthesis: definition, framework, and potential. Quality \& Quantity, 47(2), 659-676. doi: 10.1007/s11135-011-9538-6 
Hinshaw, A. D., \& Atwood, J. R. (1985). Anticipating turnover among nursing staff study: Final Report. Rockville: DHHS Division of Nursing Grant No. 1 ROl NU00908.

Hui-Ying Chiang, \& Lin, S.-Y. (2009). Psychometric testing of the Chinese version of nursing practice environment scale (Vol. 18, pp. 919-929).

Jang, E. E., McDougall, D. E., Pollon, D., Herbert, M., \& Russell, P. (2008). Integrative Mixed Methods Data Analytic Strategies in Research on School Success in Challenging Circumstances. Journal of Mixed Methods Research, 2(3), 221-247. doi: 10.1177/1558689808315323

Johnson, R. B., Onwuegbuzie, A. J., \& Turner, L. A. (2007). Toward a Definition of Mixed Methods Research. Journal of Mixed Methods Research, 1(2), 112133. doi: $10.1177 / 1558689806298224$

Kuper, A., Lingard, L., \& Levinson, W. (2008). Critically appraising qualitative research. British Medical Journal, 337, 687-689. doi: 10.1136/bmj.a1035

Lake, E. T. (2002). Development of the Practice Environment Scale of the Nursing Work Index Resarch in Nursing \& Health, 25, 176-188.

Lee, S., \& Smith, C. (2012). Criteria for Quantitative and Qualitative Data Integration. CIN: Computer, Informatics, Nursing, 30(5), 251-256. doi: 10.1097/NXN.0b013e31824b1f96

Leech, N. L., \& Onwuegbuzie, A. J. (2009). A typology of mixed methods research designs. Quality and Quantity, 43(2), 265-275. doi: http://dx.doi.org/10.1007/s11135-007-9105-3

Malina, M. A., Hanne, S. O. N., \& Selto, F. H. (2011). Lessons learned: advantages and disadvantages of mixed method research. Qualitative Research in Accounting and Management, 8(1), 59-71. doi: http://dx.doi.org/10.1108/11766091111124702

Mitchell, P. H., Ferketich, S., \& Jennings, B. M. (1998). Quality Health Outcomes Model. Journal of Nursing Scholarship, 30(1), 43-46.

Moran-Ellis, J., Alexander, V. D., Cronin, A., Dickinson, M., Fielding, J., Sleney, J., \& Thomas, H. (2006). Triangulation and integration: processes, claims and implications. Qualitative Research, 6(1), 45-59. doi: $10.1177 / 1468794106058870$

Onwuegbuzie, A. (2012). Introduction: Putting the MIXED back into quantitative and qualitative research in educational research and beyond: Moving toward the radical middle. International Journal of Multiple Research Approaches, 6(3), 192-219. doi: 10.5172/mra.2012.6.3.192

Onwuegbuzie, A., Frels, R. K., Collins, K., \& Leech, N. (2013). Conclusion: A fourphase model for teaching and learning mixed research. International Journal of Multiple Research Approaches, 7(1), 133-156. doi: 10.5172/mra.2013.7.1.133

Pearce, L. D. (2012). Mixed Methods Inquiry in Sociology. American Behavioral Scientist, 56(6), 829-848. doi: 10.1177/0002764211433798

Plano Clark, V. L. (2010). The Adoption and Practice of Mixed Methods: U.S. Trends in Federally Funded Health-Related Research. Qualitative Inquiry, 16(6), 428-440. doi: 10.1177/1077800410364609

Schmalenberg, C., \& Kramer, M. (2008). Essentials of Productive Nurse Work Environment. Nursing Research, 57(1), 2-13.

Siddiqui, N., \& Fitzgerald, A. (2009). Adaptation of quantitative research intrument to Australian Nursing Practice Environment. Paper presented at 
the ANZAM Conference 2009: Sustainable Management and Marketing, Crown Promenade, Melbourne.

Teddlie, C., \& Yu, F. (2007). Mixed methods sampling; A typology with examples Journal of Mixed Methods Research, 1(1).

Woolley, C. M. (2009). Meeting the Mixed Methods Challenge of Integration in a Sociological Study of Structure and Agency. Journal of Mixed Methods Research, 3(1), 7-25. doi: 10.1177/1558689808325774

World Health Organization. (2008). World Health Statistics 2008; Department of health statistics and informatics of the information, evidence and research cluster. WHO Library cataloguing-in-publication data.

World Health Organization. (2010). World Health Statistics 2010; Department of health statistics and informatics of the information, evidence and research cluster. WHO Library cataloguing-in-publication data. 